\title{
Características videolaringoestroboscópicas de professoras com queixas vocais
}

Videolaringoestroboscopic characteristics of female teachers with vocal complaints Características videolaringoestroboscópicas de profesores con quejas vocales

Recebido: 10/05/2020 | Revisado: 16/05/2020 | Aceito: 19/05/2020 | Publicado: 30/05/2020

\section{Daniela da Silva Gonçalves}

ORCID: https://orcid.org/0000-0002-1547-6226

Laboratório de Voz, Departamento de Fonoaudiologia, Universidade Federal de Santa Maria,

Brasil

E-mail: dani.fonors@gmail.com

Gabriele Rodrigues Bastilha

ORCID: https://orcid.org/0000-0003-4207-8001

Laboratório de Voz, Departamento de Fonoaudiologia, Universidade Federal de Santa Maria,

Brasil

E-mail: fonogabriele@gmail.com

Mara Keli Christmann

ORCID: https://orcid.org/0000-0001-8777-3660

Universidade do Vale do Itajaí e Associação Educacional Luterana Bom Jesus, Brasil E-mail: marakchristmann@gmail.com

Fabrício Scapini

ORCID: https://orcid.org/0000-0001-9975-2875

Departamento de Cirurgia, Universidade Federal de Santa Maria, Brasil

E-mail: fabricio@drfabricio.com

Carla Aparecida Cielo

ORCID: https://orcid.org/0000-0002-7219-0427

Laboratório de Voz, Departamento de Fonoaudiologia, Universidade Federal de Santa

Maria, Brasil

E-mail: cieloca@yahoo.com.br 


\title{
Resumo
}

Objetivo: verificar as características videolaringoestroboscópicas de professoras com queixas vocais. Métodos: 32 professoras com média de idade de 39,15 anos passaram por exame médico de videolaringoestroboscopia. As videolaringoestroboscopias foram avaliadas em consenso e simultaneamente por três juízes otorrinolaringologistas por meio de protocolo específico. Os dados foram analisados descritivamente e pelo teste de Correlação de Spearman, com nível de significância de $5 \%(\mathrm{p} \leq 0,05)$. Resultados: a maioria das professoras apresentou fechamento glótico normal (65,62\%). Todas apresentaram: alta simetria de vibração das pregas vocais $(92,18 \%)$, alta amplitude de vibração da onda mucosa $(90,31 \%)$, e alta presença de onda mucosa $(90,93 \%)$. Houve correlação positiva significativa entre fechamento glótico normal e amplitude de vibração $(p=0,008)$ e entre amplitude de vibração e onda mucosa $(\mathrm{p}=0)$. Houve correlação negativa significativa entre fechamento glótico alterado e amplitude de vibração ( $\mathrm{p}=0,003)$; entre fechamento glótico alterado e fechamento normal $(\mathrm{p}=0)$; e entre simetria de vibração e constrição global do vestíbulo laríngeo $(\mathrm{p}=0)$. Conclusão: a maioria das professoras, mesmo com queixas vocais e algumas com presença de nódulos vocais, apresentou fechamento glótico normal e todas apresentaram médias acima de $90 \%$ nos parâmetros: simetria de vibração das pregas vocais, amplitude de vibração da onda mucosa, e presença de onda mucosa. Neste grupo, quanto maior o fechamento glótico normal, maior a amplitude de vibração da onda mucosa, e maior a presença da onda, e quanto menor a constrição global do vestíbulo laríngeo, maior a simetria de vibração das pregas vocais.

Palavras-chave: Disfonia; Docentes; Estroboscopia; Laringoscopia; Voz.

\begin{abstract}
Objective: to verify the videolaryngostroboscopic characteristics of teachers with vocal complaints. Methods: 32 teachers with a mean of 39.15 years old underwent a medical examination of videolaryngostroboscopy. Videolaryngostroboscopies were evaluated by consensus and simultaneously by three otorhinolaryngologists using a specific protocol. Data were analyzed descriptively and by Spearman's Correlation test, with a significance level of $5 \%(\mathrm{p} \leq 0.05)$. Results: most teachers had normal glottic closure $(65.62 \%)$. All of them presented: high symmetry of vocal fold vibration (92.18\%), high amplitude of mucosal wave vibration $(90.31 \%)$, and high presence of mucous wave $(90.93 \%)$. There was a significant positive correlation between normal glottic closure and amplitude of vibration $(p=0.008)$ and between amplitude of vibration and mucosal wave $(\mathrm{p}=0)$. There was a significant negative
\end{abstract}


correlation between altered glottic closure and amplitude of vibration $(\mathrm{p}=0.003)$; between altered glottic closure and normal closure $(\mathrm{p}=0)$; and between symmetry of vibration and global constriction of the laryngeal vestibule $(\mathrm{p}=0)$. Conclusion: the majority of teachers, even with vocal complaints and some with the presence of vocal nodules, had normal glottal closure and all had averages above $90 \%$ in the parameters: symmetry of vocal fold vibration, amplitude of mucosal wave vibration, and presence of mucous wave. In this group, the greater the normal glottic closure, the greater the amplitude of vibration of the mucous wave, and what the greater the presence of the wave, and the less the global constriction of the laryngeal vestibule, the greater the symmetry of vocal fold vibration.

Keywords: Dysphonia; Laringoscopy; Stroboscopy; Teachers; Voice.

\section{Resumen}

Objetivo: verificar las características videolaringostroboscópicas de profesores con quejas vocales. Métodos: 32 profesores con una media de edad de 39,15 años se sometieron a un examen médico de videolaringostroboscopia. Las videolaringostroboscopias fueron evaluadas por consenso y simultáneamente por tres otorrinolaringólogos utilizando un protocolo específico. Los datos se analizaron descriptivamente y mediante la prueba de correlación de Spearman, con un nivel de significación del 5\% $(\mathrm{p} \leq 0,05)$. Resultados: la mayoría de los profesores tenían un cierre glótico normal $(65,62 \%)$. Todos ellos presentaron: alta simetría de la vibración de las cuerdas vocales $(92,18 \%)$, alta amplitud de la vibración de la onda mucosa $(90,31 \%)$ y alta presencia de onda mucosa $(90,93 \%)$. Hubo una correlación positiva significativa entre el cierre glótico normal y la amplitud de la vibración $(\mathrm{p}=0,008)$ y entre la amplitud de la vibración y la onda mucosa $(\mathrm{p}=0)$. Hubo una correlación negativa significativa entre el cierre glótico alterado y la amplitud de la vibración ( $\mathrm{p}=0,003)$; entre cierre glótico alterado y cierre normal $(\mathrm{p}=0)$; y entre simetría de vibración y constricción global del vestíbulo laríngeo $(\mathrm{p}=0)$. Conclusión: la mayoría de los profesores, incluso con quejas vocales y algunos con la presencia de nódulos vocales, tenían un cierre glótico normal y todos tenían promedios superiores al 90\% en los parámetros: simetría de la vibración de las cuerdas vocales, amplitud de la vibración de la onda mucosa y presencia onda mucosa En este grupo, cuanto mayor es el cierre glótico normal, mayor es la amplitud de la vibración de la onda mucosa y mayor es la presencia de la onda, y cuanto menor es la constricción general del vestíbulo laríngeo, mayor es la simetría de la vibración de las cuerdas vocales.

Palabras-clave: Disfonía; Estroboscopia; Laringoscopia; Profesores; Voz. 


\section{Introdução}

A voz é um atributo de natureza multidimensional dos seres humanos (Brasoloto \& Rehder, 2011; Halawa, García, \& Peréz, 2013; Lopes, Cavalcante, \& Costa, 2014) que envolve aspectos anatomofisiológicos, psicossociais e ambientais (Pastana, Gomes, \& Castro, 2007; Lopes et al., 2014). Qualquer alteração em um desses aspectos poderá desencadear uma disfonia (Pastana et al., 2007). Assim, a disfonia poderá ser resultado de alterações da fisiologia laríngea (Sulica, 2013).

Os distúrbios vocais necessitam de avaliações multiprofissionais (Pastana et al., 2007) que envolvam análises vocais perceptivoauditivas, acústicas, aerodinâmicas, videolaringoestroboscópicas, e avaliações da autopercepção vocal (Brasoloto \& Rehder, 2011; Pereira, Silvério, Marques, \& Camargo, 2011; Serey \& Araya, 2013; Fu, Theodoros, \& Ward, 2014). Em relação à avaliação dos aspectos anatomofisiológicos de um paciente disfônico, o exame preconizado em pesquisas na área de voz é a videolaringoestroboscopia, realizada pelo médico otorrinolaringologista (ORL) (Pastana et al., 2007; Pereira et al., 2011; Sataloff et al., 2012; Halawa et al., 2013; Serey \& Araya, 2013; Fu et al., 2014).

A videolaringoestroboscopia permite maior precisão no diagnóstico laringológico e definição do grau de severidade da patologia laríngea, uma vez que mostra com detalhes o movimento vibratório da mucosa das pregas vocais (Pastana et al., 2007; Brasoloto \& Rehder, 2011; Pereira et al., 2011; Sataloff et al., 2012; Halawa et al., 2013; Fu et al., 2014). A luz estroboscópica cria uma imagem representativa do ciclo vibratório, porém em câmera lenta, permitindo maior análise do movimento muco-ondulatório (Brasoloto \& Rehder, 2011; Pastana et al., 2007; Sulica, 2013), e suas alterações como assimetrias vibratórias, efeito de massa, fendas, dentre outras alterações estruturais (Pastana et al., 2007; Halawa et al., 2013; Sulica, 2013).

Alguns aspectos devem ser observados na avaliação videolaringoestroboscópica das pregas vocais, que são: simetria, periodicidade/regularidade de vibração ciclo a ciclo, fechamento glótico, amplitude de vibração e movimentação da onda mucosa (Pastana et al.,2007; Brasoloto \& Rehder, 2011). A gravação das imagens do exame permite comparações inter e intrassujeitos (Brasoloto \& Rehder, 2011; Sulica, 2013; Brunings et al., 2017), direcionamento de condutas terapêuticas, e comparação de resultados terapêuticos (Brasoloto \& Rehder, 2011; Sulica, 2013).

Em pesquisas na área de Voz, a utilização da videolaringoestroboscopia apontou melhora do fechamento glótico (Andrade, Cielo, Schwarz, \& Ribeiro 2016), da onda mucosa 
das pregas vocais (Niebudek-Bogusz et al., 2008; Andrade et al., 2016), da linearidade da borda livre (Niebudek-Bogusz et al., 2008), da amplitude de vibração (Niebudek-Bogusz et al., 2008; Christmann et al., 2017), reabsorção parcial de nódulos vocais (Andrade et al., 2016), redução da constrição medial do vestíbulo laríngeo (Christmann et al., 2017), redução do nível de pressão sonora e menor esforço vocal (Lima, Cielo, \& Scapini, 2015), após aplicação de diferentes técnicas vocais.

Considerando-se a capacidade de análise das modificações fisiológicas da laringe oferecida pela videolaringoestroboscopia (Pastana et al., 2007; Pereira et al., 2011; LimaSilva, Ferreira, Oliveira, Silva, \& Ghirardi, 2012; Sataloff et al., 2012; Halawa et al., 2013; Sulica, 2013; Fu et al., 2014; Fu, Theodoros, \& Ward, 2015; Brunings et al., 2017) e que os professores são o grupo de profissionais da voz com maior suscetibilidade e incidência de distúrbios vocais de ordem ocupacional (Lima-Silva et al., 2012; Serey \& Araya, 2013; Ferreira et al., 2016), o objetivo do presente estudo foi verificar as características videolaringoestroboscópicas de professoras com queixas vocais.

\section{Métodos}

Estudo transversal, observacional e quantitativo, previamente aprovado pelo Comitê de Ética em Pesquisa com Seres Humanos da instituição de origem (23081.016945/2010-76). A população-alvo foi de professoras atuantes em todos os níveis de ensino de uma cidade de médio porte. Para constituir a amostra, foram estabelecidos os seguintes critérios de inclusão: ser professor, do sexo feminino, devido à predominância na profissão docente e apresentar maior prevalência de problemas vocais em relação aos homens (Timmermans et al., 2011; Giannini, Latorre, \& Ferreira, 2012; Anhaia et al., 2014; Cielo, Ribeiro, \& Bastilha, 2015); atuar no ensino infantil, fundamental, médio ou superior das redes públicas ou privadas; ser adulta entre 19 e 60 anos (Stepp, Merchant, Heaton, \& Hillman, 2011; Bodt, Clement, Wuyts, Borghs, \& Van Lierde, 2012; Guzmán et al., 2013; Lopes et al., 2014; Fu et al., 2014; Cielo et al., 2015; Andrade et al., 2016); ser diagnosticada por médico ORL com alguma das seguintes condições laríngeas: laringe normal; fendas triangulares grau I ou II, fendas fusiformes (causadas por fadiga muscular secundária à hipertensão), hiperconstrição supraglótica, alterações organofuncionais como nódulos vocais ou outras; relatar queixas vocais (Rodriguez-Parra, Adian, \& Casado, 2011; Stepp et al., 2011; Watts et al., 2014; Wenke et al., 2014); e carga horária de trabalho semanal igual ou superior a 20 horas. 
Os critérios de exclusão foram: estar em período de licença, trabalhar em salas de apoio ou atividades administrativas, devido à demanda vocal menor e ou menos intensa (Marçal \& Peres, 2011; Giannini et al., 2012); histórico de doenças neurológicas, endocrinológicas, psiquiátricas, gástricas ou respiratórias crônicas (Dagli et al., 2008; Lopes et al., 2014; Wenke et al., 2014; Fu et al., 2014; Fu et al., 2015; Cielo et al., 2015; Andrade et al., 2016; Menezes et al., 2020); relato de gravidez, período menstrual ou pré-menstrual (em torno de cinco dias antes do início da menstruação), gripe ou qualquer alergia respiratória nos dias das avaliações e reavaliações, condições que podem vir a causar edema temporário nas pregas vocais (Wenke et al., 2014; Andrade et al., 2016; Menezes et al., 2020); relato de etilismo e/ou tabagismo (Cielo et al., 2015; Andrade et al., 2016); realização de tratamento fonoaudiológico relacionado à voz ou aulas de canto prévios, a fim de evitar sujeitos com maior condicionamento vocal (Bodt et al., 2012; Fu et al., 2014; Fu et al.,2015; Menezes et al., 2020); perda auditiva (Dagli et al., 2008; Stepp et al., 2011; Rodriguez-Parra et al., 2011; Bodt et al., 2012; Wenke et al., 2014; Fu et al., 2014; Fu et al., 2015; Andrade et al., 2016; Menezes et al., 2020).

Foram realizadas entrevista e triagem auditiva (audiômetro Fonix FA 12 Digital, Frye Electronics, Estados Unidos). A videolaringoestroboscopia foi realizada por um médico ORL (aparelho Atmos Lenzkirch, óptica Storz de $70^{\circ}$ - Tuttlingen, Alemanha), as professoras emitiram as vogais /e/, /i/ e fonação reversa com leve inclinação anterior e para cima da cabeça (Rodriguez-Parra et al., 2011; Fu et al., 2015; Fu et al., 2014; Pedrosa, Pontes, Pontes, Behlau, \& Peccin, 2015; Menezes et al., 2020).

Após a avaliação médica, uma professora foi excluída por doença do refluxo gastroesofágico, uma pela impossibilidade de visualização das pregas vocais durante o exame, e uma devido à intensa assimetria do complexo aritenoideo. Participaram, por fim, 32 professoras, com idades entre 24:8 e 59:7 anos (média 39:15 anos), sendo 12 com presença de nódulos vocais e o restante sem lesão laríngea.

Os exames videolaringoestroboscópicos foram editados, codificados, randomizados e gravados em pen drive para serem avaliados por ORL juízes. Os aspectos avaliados com base em uma escala analógico-visual foram: amplitude de vibração da mucosa, constrição do vestíbulo laríngeo (global, medial e ântero-posterior), simetria da vibração das pregas vocais, e presença de onda mucosa. Na avaliação do tipo de fechamento glótico, os juízes deviam apontar a ocorrência de umas das seguintes opções em cada exame avaliado: fechamento glótico completo, fenda triangular grau I, fenda triangular grau II, fenda triangular em toda 
extensão, fenda em ampulheta, fenda fusiforme, fenda em duplo fuso e fenda fusiforme parcial (Halawa et al., 2013; Fu et al., 2014; Pedrosa et al., 2015).

Para análise dos resultados, o fechamento glótico foi dividido, em: "normal" e "alterado". O fechamento glótico normal incluiu o fechamento glótico completo e a fenda triangular grau I, comum na população feminina e considerada uma inadaptação fônica, sem interferência na fonação (Dedivitis, 2002; Cielo, Schwarz, Finger, Lima, \& Christmann, 2019). O fechamento foi considerado "alterado" para outros tipos de fendas com ou sem presença de patologias laríngeas.

A avaliação das imagens de videolaringoestroboscopia foi realizada em consenso e simultaneamente por três juízes ORL (Lopes et al., 2014), com experiência em laringologia de no mínimo cinco anos. A escala analógico-visual utilizada apresentava $10 \mathrm{~cm}$ de extensão (de zero a $100 \mathrm{~mm}$ ); a marcação considerava a extremidade esquerda (zero) como ausência total do parâmetro avaliado e a direita como o grau máximo do parâmetro em questão (RodriguezParra et al., 2011; Lopes et al., 2014; Pedrosa et al., 2015).

Após as avaliações dos juízes, a marcação da escala analógico-visual foi transformada em $\mathrm{mm}$, por meio de leitura direta com régua milimetrada, convertendo-se a um valor percentual para a análise estatística dos dados. Todos os juízes foram cegados quanto aos objetivos, a metodologia da pesquisa, a identificação das professoras, e quanto aos resultados da análise do médico que realizou os exames iniciais para os critérios de inclusão (RodriguezParra et al., 2011; Giannini et al., 2012; Cielo \& Christmann, 2014).

Os dados foram analisados estatisticamente através de análise descritiva e Correlação de Spearman, com nível de significância de $5 \%(\mathrm{p} \leq 0,05)$.

\section{Resultados}

Na Tabela 1, estão expostos os resultados da análise descritiva das características videolaringoestroboscópicas deste grupo de professoras com queixas vocais. Houve maiores resultados de fechamento glótico normal, de amplitude de vibração da mucosa, de simetria de vibração das pregas vocais, e de presença de onda mucosa. 
(CC BY 4.0) | ISSN 2525-3409 | DOI: http://dx.doi.org/10.33448/rsd-v9i7.4397

Tabela 1 - Análise descritiva das características laríngeas de professoras com queixas vocais através da videolaringoestroboscopia (Santa Maria/RS, 2020).

\section{Características laríngeas videolaringoestroboscópicas}

\begin{tabular}{|c|c|c|c|c|c|c|c|}
\hline & $\begin{array}{c}\text { Média } \\
(\%)\end{array}$ & $\begin{array}{c}\text { Mediana } \\
(\%)\end{array}$ & $\begin{array}{c}\text { Mínimo } \\
(\%)\end{array}$ & $\begin{array}{c}\text { Máximo } \\
(\%)\end{array}$ & DP & $\mathbf{n}$ & $\%$ \\
\hline $\begin{array}{l}\text { Fechamento } \\
\text { glótico } \\
\text { normal }\end{array}$ & 31,25 & 0 & 0 & 100 & 47,09 & 11 & 65,62 \\
\hline $\begin{array}{l}\text { Fechamento } \\
\text { glótico } \\
\text { alterado }\end{array}$ & 65,62 & 100 & 0 & 100 & 48,25 & 21 & 34,37 \\
\hline $\begin{array}{l}\text { Amplitude } \\
\text { de vibração } \\
\text { da mucosa }\end{array}$ & 90,31 & 100 & 50 & 100 & 12,30 & 32 & 100 \\
\hline $\begin{array}{l}\text { Constrição } \\
\text { medial do } \\
\text { vestíbulo } \\
\text { laríngeo }\end{array}$ & 9,37 & 0 & 0 & 100 & 27,81 & 4 & 12,50 \\
\hline $\begin{array}{l}\text { Constrição } \\
\text { ântero- } \\
\text { posterior do } \\
\text { vestíbulo } \\
\text { laríngeo }\end{array}$ & 1,56 & 0 & 0 & 50 & 8,83 & 1 & 3,12 \\
\hline $\begin{array}{l}\text { Constrição } \\
\text { global do } \\
\text { vestíbulo } \\
\text { laríngeo }\end{array}$ & 6,25 & 0 & 0 & 100 & 21,06 & 3 & 9,37 \\
\hline $\begin{array}{l}\text { Simetria da } \\
\text { vibração } \\
\text { das pregas } \\
\text { vocais }\end{array}$ & 92,18 & 100 & 0 & 100 & 20,11 & 32 & 100 \\
\hline $\begin{array}{l}\text { Presença de } \\
\text { onda } \\
\text { mucosa }\end{array}$ & 90,93 & 100 & 50 & 100 & 12,27 & 32 & 100 \\
\hline
\end{tabular}

Legenda: DP = desvio-padrão; $\mathrm{n}$ = número de sujeitos.

A Tabela 2 apresenta as correlações entre os parâmetros da videolaringoestroboscopia. Houve correlação significativa positiva entre amplitude de vibração da mucosa e fechamento glótico normal e entre amplitude de vibração da mucosa e presença de onda mucosa. Houve 
correlação significativa negativa entre fechamento glótico alterado e amplitude de vibração da mucosa; entre fechamento glótico alterado e fechamento glótico normal; e entre simetria de vibração das pregas vocais e constrição global do vestíbulo laríngeo.

Tabela 2 - Correlação entre os parâmetros da videolaringoestroboscopia de professoras com queixas vocais (Santa Maria/RS, 2020).

Variáveis correlacionadas

\section{Fechamento}

glótico normal
Fechamento glótico alterado

Amplitude de vibração da mucosa

Constrição medial do vestíbulo laríngeo

Constrição ântero-posterior do vestíbulo laríngeo

Constrição global do vestíbulo laríngeo

Simetria da vibração das pregas vocais

Presença de onda mucosa

Amplitude de vibração da mucosa

Constrição medial do vestíbulo laríngeo

Constrição ântero-posterior do vestíbulo laríngeo

Constrição global do vestíbulo laríngeo

Simetria da vibração das pregas vocais

Presença de onda mucosa

Constrição medial do vestíbulo laríngeo

Constrição ântero-posterior do vestíbulo laríngeo

\section{Corr(r) p-valor}

$-0,931 \quad 0^{*}$

$0,457 \quad 0,008^{*}$

$-0,057 \quad 0,755$

$-0,121 \quad 0,509$

$0,028 \quad 0,875$

$0,050 \quad 0,783$

$0,288 \quad 0,108$

$-0,504 \quad 0,003^{*}$

$0,080 \quad 0,660$

$0,129 \quad 0,478$

$-0,007 \quad 0,969$

$-0,084 \quad 0,647$

$-0,335 \quad 0,060$

\begin{tabular}{llll}
\hline $\begin{array}{l}\text { Amplitude de } \\
\text { vibração da }\end{array}$ & Constrição medial do vestíbulo laríngeo & $-0,310$ & 0,083 \\
mucosa & Constrição ântero-posterior do vestíbulo & $-0,190$ & 0,297 \\
& laríngeo & &
\end{tabular}


Constrição global do vestíbulo laríngeo $\quad 0,079 \quad 0,663$

Simetria da vibração das pregas vocais $\quad 0,222 \quad 0,220$

Presença de onda mucosa $\quad 0,825 \quad 0^{*}$

\begin{tabular}{llcc}
\hline $\begin{array}{l}\text { Constrição medial } \\
\text { do vestíbulo }\end{array}$ & Constrição ântero-posterior do vestíbulo & $-0,067$ & 0,712 \\
laríngeo & laríngeo & & \\
& Constrição global do vestíbulo laríngeo & $-0,121$ & 0,508 \\
& Simetria da vibração das pregas vocais & $-0,278$ & 0,122 \\
& Presença de onda mucosa & $-0,164$ & 0,368 \\
\hline $\begin{array}{l}\text { Constrição ântero- } \\
\text { posterior do }\end{array}$ & Constrição global do vestíbulo laríngeo & $-0,057$ & 0,753 \\
vestíbulo laríngeo & Simetria da vibração das pregas vocais & 0,094 & 0,607 \\
& Presença de onda mucosa & $-0,203$ & 0,263 \\
\hline $\begin{array}{l}\text { Constrição global } \\
\text { do vestíbulo }\end{array}$ & Simetria da vibração das pregas vocais & $-0,555$ & $0 *$ \\
laríngeo & Presença de onda mucosa & 0,060 & 0,742 \\
\hline $\begin{array}{l}\text { Simetria da } \\
\text { vibração das }\end{array}$ & Presença de onda mucosa & & \\
pregas vocais & & 0,252 & 0,162 \\
\hline
\end{tabular}

*Correlação de Spearman $(\mathrm{p} \leq 0,05)$

Legenda: Corr $=$ correlação

\section{Discussão}

$\mathrm{Na}$ amostra de 32 professoras com queixas vocais deste estudo, apesar da presença de nódulos vocais em 12 delas, o aspecto simetria de vibração das pregas vocais obteve uma avaliação média de 92,18\% (Tabela 1). Os movimentos vibratórios das pregas vocais são considerados simétricos quando o tempo de abertura, de fechamento, ou a extensão da excursão lateral durante a vibração parecem iguais para as duas pregas vocais, o que indica 
que suas propriedades mecânicas são as mesmas durante o processo de fonação (Hirano \& Bless, 1997).

Para explicar o mecanismo neurofisiológico da hiperfunção da laringe durante a fonação, estudo comparou um grupo de mulheres saudáveis e um de mulheres disfônicas. Das 10 mulheres com disfonia por tensão muscular, 60\% apresentaram assimetria e $70 \%$ onda mucosa reduzida (Kryshtopava et al., 2017), diferindo dos achados do presente estudo.

Convergindo com nossos resultados, em pesquisa com 70 indivíduos diagnosticados com nódulos vocais, o exame videolaringoestroboscópico revelou maior frequência dessa afecção em adultos do sexo feminino e professores. E apontou pregas vocais com vibrações simétricas ou quase simétricas mesmo na presença de nódulos vocais. O estudo citado ressalta que não existe relação direta entre simetria laríngea e voz normal e entre assimetria laríngea e voz alterada; e que é comum que alguns indivíduos com simetria laríngea possam apresentar soprosidade, rouquidão, frequências fora da sua gama tonal, fadiga vocal, e/ou até mesmo lesões de massa (Braga, Oliveira, Atherino, Schott, \& Silva, 2006).

Segundo a avaliação dos juízes ORL, a segunda maior média do grupo de professoras estudado foi referente à presença de onda mucosa (90,93\%) (Tabela 1). Um dos traços mais importantes da vibração das pregas vocais é a ocorrência de ondas que percorrem a mucosa da superfície inferior à superior da prega vocal (Hirano \& Bless, 1997). A presença da onda mucosa pode ser interpretada como uma evidência da integridade viscoelástica em relação ao corpo da prega vocal (Hirano \& Bless, 1997; Dedivitis, 2002).

O aumento da onda mucosa comumente ocorre na emissão de frequências baixas, em situações hiperfuncionais (Dedivitis, 2002), em lesões edematosas (Hirano \& Bless, 1997), e em casos de aumento da pressão aérea subglótica (Hirano \& Bless, 1997; Dedivitis, 2002). Essas situações estão de acordo com o grupo de professoras estudado que relatou queixas vocais, sendo que 12 delas apresentavam nódulos vocais que são lesões de característica localmente edematosa.

Condições fisiológicas ou patológicas podem diminuir a amplitude de vibração das pregas vocais como o encurtamento da sua porção vibrante, menor flexibilidade (fonação em frequência fundamental alta), presença de lesão de massa (Hirano \& Bless, 1997; Dedivitis, 2002). Nesta pesquisa, as professoras obtiveram a média de 90,31\% para a amplitude de vibração das pregas vocais (Tabela 1). Tais resultados divergem de estudos em que, para 70\% de mulheres com disfonia (Kryshtopava et al., 2017) e para uma professora com queixa vocal de fadiga e afonia ao final do dia (Pastana et al., 2007), a amplitude de vibração se apresentou reduzida. 
As correlações significativas positivas neste estudo se deram entre amplitude de vibração da mucosa e fechamento glótico normal e entre amplitude de vibração e presença de onda mucosa (Tabela 2), mostrando que quanto mais adequado é o fechamento glótico maior é a amplitude de vibração e presença de onda mucosa. O que está de acordo com as médias porcentuais de amplitude de vibração da mucosa, presença de onda mucosa e de simetria de vibração das pregas vocais que se apresentaram acima de 90\% (Tabela 1).

Pôde-se observar que, quanto menos alterado foi o fechamento glótico, maior foi a amplitude de vibração das pregas vocais. Outra referência a esses parâmetros foi encontrada em casos com diagnóstico de disfonia por tensão muscular, em que as porcentagens referentes à presença de fechamento glótico incompleto $(80 \%)$ e a presença de amplitude de vibração da mucosa reduzida (70\%) foram aproximadas (Kryshtopava et al., 2017).

Salienta-se que os parâmetros de avaliação da videolaringoestroboscopia devem ser analisados em conjunto e complementados por avaliação fonoaudiológica, uma vez que mecanismos compensatórios podem estar presentes proporcionando um fechamento glótico completo, mas à custa de uma de qualidade vocal tensa (Dedivitis, 2002).

Situações fisiológicas devem ser reconhecidas, como a presença de pequena fenda triangular grau I. Este tipo de fenda é considerado "normal" no sexo feminino, uma vez que se limita à glote intercartilagínea não se estendendo ventralmente ao processo vocal das aritenoides (Dedivitis, 2002). Portanto, a aproximação das cartilagens aritenoides não é completa, criando uma fenda glótica posterior à fonação restrita à área respiratória, não interferindo na produção vocal (Cielo et al., 2019).

Uma mucosa flexível é essencial para a ocorrência adequada da onda mucosa das pregas vocais (Hirano \& Bless, 1997). Quanto maior a onda mucosa, maior a amplitude de vibração (Dedivitis, 2002), conforme mostraram nossos resultados (Tabela 2). Na proporcionalidade desses parâmetros, obtém-se um sinal glótico mais periódico, com menor ruído e maior número de harmônicos. O fechamento glótico alterado e a amplitude de vibração foram os parâmetros que apresentaram correlação significativa negativa no grupo de professoras estudado (Tabela 2).

A literatura evidencia a grande ocorrência de lesões benignas das pregas vocais em docentes (Braga et al., 2006; Woźnicka, Niebudek-Bogusz, Morawiska, \& ŚliwińskaKowalska, 2017; Mobarsa, Samdani, \& Gurjar, 2018; Vivek, Sabah, Nidhi, \& Neethu, 2018) do sexo feminino (Braga et al., 2006; Banjara, Mungutwar, Singh, Gupta, \& Singh, 2012; Mobarsa et al., 2018). O nódulo vocal é a lesão mais frequente (Banjara et al., 2012; Woźnicka et al., 2017; Mobarsa et al., 218; Vivek, et al., 2018) e o tipo mais comum de 
fechamento glótico encontrado na presença de nódulos foi o padrão de fenda em ampulheta (Banjara et al., 2012; Vivek, et al., 2018). Isto converge com nossos achados em que, das 32 professoras, 12 apresentavam nódulos vocais.

Neste trabalho, houve correlação significativa negativa entre simetria de vibração (alto percentual) e constrição global do vestíbulo laríngeo (baixo percentual), mostrando que quanto maior a simetria de vibração, menor a constrição global do vestíbulo laríngeo (Tabelas 1 e 2). A contrição do vestíbulo laríngeo pode promover diferenças nas propriedades mecânicas das pregas vocais resultando em vibrações assimétricas, com piora da qualidade vocal (Dedivitis, 2002; Hirano \& Bless, 1997). A presença de nódulos vocais, dentre outras patologias, apresenta relação estatisticamente significativa com a simetria de vibração, pois essa lesão geralmente envolve ambas as pregas vocais (Banjara et al., 2012). Tais afirmações convergem parcialmente com nossos resultados, onde, sempre que a constrição global do vestíbulo laríngeo foi reduzida, a simetria de vibração das pregas vocais foi aumentada, mesmo com $12(37,5 \%)$ das professoras apresentando nódulos vocais.

Em pesquisa, a constrição supraglótica foi observada em 47 cantores profissionais saudáveis (Castelblanco et al., 2014). Em mulheres com disfonia por tensão muscular, as porcentagens de constrição medial e constrição anteroposterior do vestíbulo laríngeo foram semelhantes (Kryshtopava et al., 2017). E em um grupo de 31 estudantes de teatro musical, cuja avaliação videolaringoestroboscópica apontou constrição supraglótica durante a fonação habitual, não ficou claro se a constrição se referiu a uma hiperfunção ou estratégia de canto para aumento de projeção vocal (D'haeseleer et al., 2016).

Através da análise geral dos parâmetros videolaringoestroboscópicos, as professoras com queixas vocais apresentaram como características principais previamente discutidas: o fechamento glótico normal, grande simetria de vibração das pregas vocais, grande amplitude de vibração da mucosa, e alta presença de onda de mucosa das pregas vocais, apesar de 37,5\% das professoras estudadas terem apresentado nódulos vocais.

Tendo em vista o caráter multidimensional da voz, sugere-se que estudos futuros correlacionem os parâmetros da videolaringoestroboscopia, com as avaliações acústicas, perceptivoauditivas, aerodinâmicas e de autopercepção vocal, visando auxiliar na conduta terapêutica adequada e na prevenção de distúrbios vocais e laríngeos, favorecendo o diagnóstico precoce. 


\section{Conclusão}

Os professores são considerados o grupo de profissionais da voz com maior suscetibilidade e incidência de distúrbios vocais de ordem ocupacional com grande ocorrência de lesões benignas das pregas vocais. Isto se confirmou parcialmente uma vez que, no grupo analisado, a avaliação médica videolaringoestroboscópica revelou que a maioria das professoras, mesmo com queixas vocais e algumas com presença de nódulos vocais, apresentou fechamento glótico normal e que todas as professoras apresentaram médias elevadas nos parâmetros: simetria de vibração das pregas vocais, amplitude de vibração da onda mucosa, e presença de onda mucosa.

Neste grupo, quanto maior o fechamento glótico normal, maior a amplitude de vibração da onda mucosa, que diminuiu quando o fechamento glótico foi alterado. Quanto maior a amplitude de vibração da onda mucosa, maior a presença de onda mucosa, e quanto menor a constrição global do vestíbulo laríngeo, maior a simetria de vibração das pregas vocais. Tais conclusões apontam para uma funcionalidade laríngea melhor do que a esperada, considerando-se a presença de queixas vocais e lesões de pregas vocais no grupo avaliado.

\section{Agradecimentos}

O presente trabalho foi realizado com apoio da Coordenação de Aperfeiçoamento de Pessoal de Nível Superior - Brasil (CAPES) - Código de Financiamento 001 e Conselho Nacional de Desenvolvimento Científico e Tecnológico (CNPQ) (Grant 301326/2017-7).

\section{Referências}

Andrade, S.R., Cielo, C.A., Schwarz, K., \& Ribeiro, V.V. (2016). Vocal therapy and nasal sounds: effects on hyperfunctional dysphonia. CEFAC - Speech, Language, Hearing Sciences and Education Journal, 18(1), 263-272.

Anhaia, T.C., Klahr, P.S., Ourique, A.A.B., Gadenz, C.D., Fernandes, R.A., Spagnol, P.E., ... Cassol, M. (2014). Effects of two interventions in teachers with voice complaints. Audiology Communication Research, 19(2), 186-193. 
Banjara, H., Mungutwar, V., Singh, D., Gupta, A., \& Singh, S. (2012). Demographic and videostroboscopic assessment of vocal pathologies. Indian Journal of Otolaryngology and Head Neck Surgery, 64(2), 150-157.

Bodt, M.D., Clement, G., Wuyts, F., Borghs, C., \& Van Lierde, K.M. (2012). The impact of phonation mode and vocal technique on vocal fold closure in young females with normal voice quality. Journal of Voice, 26(6), 818.e1-818.e4.

Braga, J.N., Oliveira, D.S.F., Atherino, C.C.T., Schott, T.C.A., \& Silva, J.C. (2006). Vocal nodules: functional and anatomical analysis. CEFAC - Speech, Language, Hearing Sciences and Education Journal, 8(2), 223-229.

Brasolotto, A.G., \& Rehder, M.I. (2011). Diagnóstico vocal fonoaudiológico. In: Rehder, M.I., \& Branco, A.A.O. (Org.). Disfonia e Disfagia: interface, atualização e prática. Rio de Janeiro: Revinter, 6-7.

Brunings, J.W., Vanbelle, S., Akkermans, A., Heesmskerk, N.M.M., Kremmer, B., Stokroos, R.J., \& Baijens, L.W.J. (2017). Observer agreement for measurements in videolaryngostroboscopy. Journal of Voice, 32(6), 756-762.

Castelblanco, L., Habib, M., Stein, D.J., Quadros, A., Cohen, S.M., \& Noordzij, J.P. (2014). Singing voice handicap and videostrobolaryngoscopy in healthy professional singers. $\underline{\text { Journal }}$ of Voice, 28(5), 608-613.

Chistmann, M.K, Gonçalves, B.F.T, Lima, J.P.M, Bastilha, G.R, Scapini, F., \& Cielo, C.A. (2017). Short and intensive therapy with finger kazoo in patient with organic dysphonia postorotracheal intubation. Revista Distúrbios da Comunicação, 29(1), 41-54.

Cielo, C. A., \& Christmann, M. K. (2014). Finger kazoo: spectrographic acoustic modifications and vocal self-assessment. CEFAC - Speech, Language, Hearing Sciences and Education Journal, 16(4), 1239-1254. 
Cielo, C.A. Ribeiro, V.V., \& Bastilha, G.R. (2015). Spectrographic voice measures, vocal complaints and occupational data of elementary school teachers Revista Distúrbios da Comunicação, 27(2), 299-308.

Cielo, C.A., Schwarz, K., Finger, L.S., Lima, J.M., \& Christmann, M.K. (2019). Glottal closure in women with no voice complaints or laryngeal disorders. International Archives of Otorhinolaryngology, 23(4), e384-e388.

D'haeseleer, E., Claeys, S., Meerschman, I., Bettens, K., Degeest, S., Dijckmans, ...Van Lierde, K. (2017). Vocal characteristics and laryngoscopic findings in future musical theater performers. Journal of Voice, 31(4), 462-469.

Dagli, M., Sati, I., Acar, A., Stone JR, R.E., Dursun, G., \& Eryilmaz, A. (2008). Mutational falsetto: intervention outcomes in 45 patients. The Journal of Laryngology \& Otology, 122(3), 277-281.

Dedivitis R.A. (2002). Estroboscopia. In: Dedivitis R.A, \& BarroS A.P.B, editores. (Org.). Métodos de avaliação e diagnóstico de laringe e voz. São Paulo: Lovise, 71-88.

Ferreira, L.P., Giannini, S.P.P., Alves, N.L.L., Brito, A.L., Andrade, B.M.R., \& Latorre, M.R.D.O. (2016). Voice disorder and teaching work ability. CEFAC - Speech, Language, Hearing Sciences and Education Journal, 18(4), 932-940.

Fillis, M.M.A., Andrade, S.M., González, A.D., Melanda, F.N., \& Mesas, A.E. (2016). Frequency of self-reported vocal problems and associated occupational factors in primary schoolteachers in Londrina, Paraná State, Brazil. Cadernos de Saúde Pública, 32(1), e00026015.

Fu, S., Theodoros, D.G., \& Ward, E.C. (2015). Delivery of intensive voice therapy for vocal fold nodules via telepractice: A pilot feasibility and efficacy study. Journal of Voice, 29(6), 696-706. 
Fu, S., Theodoros, D. G., \& Ward, E. C. (2014). Intensive versus traditional voice therapy for vocal nodules: perceptual, physiological, acoustics and aerodynamic changes. Journal of Voice, 29(2), 260. p. e31-260.e44.

Giannini, S.P.P., Latorre, M.R.D.O., \& Ferreira, L. P. (2012). Distúrbio de voz e estresse no trabalho docente: um estudo caso-controle. Cadernos de Saúde Pública, 28(11), 2115-2124.

Guzmán, M., Laukkanen, A., Krupa, P., Horáček, J., 占vec, J.G., \& Geneid, A. (2013). Vocal tract and glottal function during and after vocal exercising with resonance tube and straw. Journal of Voice, 27(4), 523.e19-523.e34.

Halawa, W.E., Garcia, A.C., \& Perez, S.S. (2013). Effectiveness of laryngostroboscopy for monitoring the evolution of vocal nodules after rehabilitator treatment. Auris Nasus Larynx, 40(1), 204-206.

Hirano, M. \& Bless, D.M. Julgamento e interpretação do padrão vibratório. In: Hirano, M., \& Bless, D.M. Exame videoestroboscópico da laringe. São Paulo: Artes Médicas, 125-134.

Kryshtopava, M., Van Lierde, K., Meerschman, I., D’haeseleer, E., Vandemaele, P., Vingerhoets, G., \& Claeys, S. (2017). Brain activity during phonation in women with muscle tension dysphonia: An FMRI study. Journal of Voice, 31(6), 675-690.

Lima, J.P.M., Cielo, C.A., \& Scapini, F. (2015). Phonation into a glass tube immerse in water: analysis perceptiveauditory vocal and videolaryngostroboscopy in women without laryngeal disorders, complaints or vocal alterations. CEFAC - Speech, Language, Hearing Sciences and Education Journal, 17(6), 1760-1772.

Lima-Silva, M.F.B., Ferreira, L.P., Oliveira, I.B., Silva, M.A.A., \& Ghirardi, A.C.A.M. (2012). Voice disorders in teachers: self-report, auditory-perceptive assessment of voice and vocal fold assessment. Revista Sociedade Brasileira de Fonoaudiologia, 17(4), 391-397.

Lopes, L.W., Cavalcante, D.P., \& Costa. P.O. (2014). Severity of voice disorders: integration of perceptual and acoustic data in dysphonic patients. Communication Disorders, Audiology and Swallowing, 26(5), 382-388. 
Luchesi, K.F., Mourão, L.F., \& Kitamura, S. (2012). Effectiveness of a vocal improvement program for teachers. CEFAC - Speech, Language, Hearing Sciences and Education Journal, 14(3), 459-470.

Marçal, C.C.B., \& Peres, M.A. (2011). Self-reported voice problems among teachers: prevalence and associated factors. Revista de Saúde Pública, 45(3), 503-511.

Maxfield, L., Titze, I., Hunter, E., \& Kapsner-Smith, M. (2015). Intraoral pressures produced by thirteen semi-occluded vocal tract gestures. Logopedics Phoniatrics Vocology, 40(2), 8692.

Menezes, E.M., Luz, T.A.P, Bastilha, G.R., Christmann, M.K., \& Cielo, C.A. Intensive shortterm vocal therapy with Finger Kazoo in male teachers - case study. Research, Society and Development, 9(7):1-18, e177973863.

Mobarsa, V., Samdani S.K., \& Gurjar, V.S. (2018). Outcome analysis of microlaryngeal surgery for benign lesions of vocal cord using videostroboscopy and Voice Handicap Index. Indian Journal Otolaryngology and Head Neck Surgery, 71 (Suppl 1), 327-332.

Niebudek-Bogusz, E., Sznurowska-Przygocka, B., _ F,P., Kotyło, M., Sinkiewicz, A., Modrzewska,_M., \& Sliwinska-Kowalska, M. (2008). The effectiveness of voice therapy for teachers with dysphonia. Folia Phoniatrica et Logopaedica, 60(3), 134-141.

Pastana, S. G., Gomes, E., \& Castro, L. (2007). Speech therapy and stroboscopy evaluation in cyst differential diagnosis. CEFAC - Speech, Language, Hearing Sciences and Education Journal, 9(3), 383-396.

Pedrosa, V., Pontes, A., Pontes, P., Behlau, M., \& Peccin, S.M. (2015). The effectiveness of the comprehensive voice rehabilitation program compared with the vocal function exercises method in behavioral dysphonia: a randomized clinical trial. Journal of Voice, 30(3), 377 e $11-9$. 
Pereira, E.C., Silvério, K.C.A., Marques, J.M., \& Camargo, P.A.M. (2011). Immediate effect of vocal techniques in women without vocal complaint. CEFAC - Speech, Language, Hearing Sciences and Education Journal, 13(5), 886-894.

Pimenta, R. A., Dájer, M.E., Hachiya, A., Cordeiro, G.F., Tsuji, D.H., \& Montagnoli, A.N. (2013). High-speed kymography identifies the immediate effects of voiced vibration in healthy vocal folds. International Archives of Otorhinolaryngology, 17(1), 74-79.

Rodriguez-Parra, M. J., Adian, J. A., \& Casado, J. C. (2011). Comparing voice-therapy and vocal-hygiene treatments in dysphonia using a limited multidimensional evaluation protocol. Journal Communication Disorders, 44(6), 615-630.

Sataloff, R.T., Hawkshaw, M.J., Johson, J.L., Ruel, B., Wilhelm, A., \& Lurie, D. (2012). Prevalence of abnormal laryngeal findings in healthy singing teachers. Journal of Voice, 26(5), 577-583.

Serey, J.P., \& Araya V.O. (2013). Instruments used in the evaluation of teachers' voice: literature review. CEFAC - Speech, Language, Hearing Sciences and Education Journal, 15(5), 1357-1363.

Stepp, C.E., Merchant, G.R., Heaton, J.T., \& Hillman, R.E. (2011). Effects of voice therapy on relative fundamental frequency during voicing offset and onset in patients with vocal hyperfunction. Journal of Speech, Language, and Hearing Research, 54(5), 1260-1266.

Sulica, L. (2013). Laryngoscopy, stroboscopy and other tools for the evaluation of voice desorders. Otolaryngologic Clinics of North America, 46(1), 21-30.

Timmermans, B., Coveliers, Y., Meeus, W., Vandenabeele, F., Van Looy, L., \& Wuyts, F. (2011). The effect of a short voice training program in future teachers. Journal of Voice. 25(4), e191-e198.

Tsuji, D.H., Hachiya, A., Dajer, M.E., Ishikawa, C.C., Takahashi, M.T., \& Montagnoli, A.N. (2014). Improvement of vocal pathologies diagnosis using high-speed videolaryngoscopy. International Archives of Otorhinolaryngology, 18(3), 294-302. 
Vivek, S., Sabah, M., Nidhi, M., \& Neethu, S.G. (2018). Study of pre and postoperative videostroboscopic evaluation of benign vocal cord lesions. Indian Journal of Otolaryngology and Head \& Neck Surgery, 71(Suppl 1), 327-332.

Watts, C.R., Diviney, S.S., Hamilton, A., Toles, L., Childs, L., \& Mau, T. (2014). The effect of stretch-and-flow voice therapy on measures of vocal function and handicap. Journal of Voice, 29(2), 191-199.

Wenke, R.J., Stabler, P., Walton, C., Coman, L., Lawrie, M., O’neill, J., ... Carde 11, E. (2014). Is more intensive better? Client and service provider outcomes for intensive versus standard therapy schedules for functional voice disorders. Journal of Voice, 28(5), 652.e31-652.e43.

Woźnicka, E., Niebudek-Bogusz, E., Morawiska, J., \& Śliwińska-Kowalska, M. (2017). Laryngeal manual therapy palpatory evaluation scale: A preliminary study to examine its usefulness in diagnosis of occupatio

\title{
Porcentagem de contribuição de cada autor no manuscrito
}

\author{
Daniela da Silva Gonçalves - 30\% \\ Gabriele Rodrigues Bastilha - 20\% \\ Fabrício Scapini - 15\% \\ Mara Keli Christmann - 15\% \\ Carla Aparecida Cielo - 20\%
}

